\title{
Crianças e adolescentes obesos: dois anos de acompanhamento interdisciplinar
}

\author{
Obese children and adolescents: two years of interdisciplinary follow-up
}

\author{
Mariana Porto Zambon', Maria Ângela R. G. M. Antonio ${ }^{1}$, Roberto Teixeira Mendes ${ }^{1}$, Antônio de Azevedo Barros Filho
}

\section{RESUMO}

Objetivo: Avaliar dois anos de seguimento de crianças e adolescentes obesos em ambulatório especializado.

Métodos: Trata-se de coorte, com coleta retrospectiva dos dados de 150 pacientes (1 a 19 anos de idade) do Ambulatório de Obesidade do Hospital de Clínicas da Universidade Estadual de Campinas. Destes pacientes, $53 \%$ eram do sexo masculino e 63\% eram de Campinas, São Paulo. A média da idade no início de ganho de peso foi 4,3 anos. Dos 150 pacientes, 128 retornaram uma vez, com avaliação de peso, altura, índice de massa corpórea (IMC) e escores Z. Foram realizados hemograma, glicemia de jejum, insulina basal, relação glicemia/insulina, triglicérides, colesterol total e frações. Os pacientes foram divididos em dois grupos, de acordo com o seguimento. Entre os grupos, foi analisada a diferença entre idade, sexo, procedência, idade de início, acantose nigricans e os escores $Z$ de peso, altura e IMC, proporção de exames laboratoriais alterados e a diferença dos escores $Z$ de IMC em relação à primeira consulta.

Resultados: Dos 128 pacientes, 57\% permaneceram em seguimento, sendo que 114 retornaram pelo menos uma vez. Comparando os grupos, os moradores de Campinas foram os que mais abandonaram o ambulatório, sem diferenças em relação às demais variáveis clínicas, antropométricas e laboratoriais estudadas. Houve queda da média do escore $\mathrm{Z}$ do IMC em ambos os grupos, em relação à consulta inicial.

Conclusões: Os pacientes deste estudo apresentaram redução da média do escore $Z$ do IMC semelhante à literatura. Apesar do alto índice de abandono, não foram identificados fatores de risco que o justificassem, com exceção da procedência da criança.
Palavras-chave: obesidade; antropometria; testes hematológicos; criança; adolescente.

\section{ABSTRACT}

Objective: To study two years follow-up of obese children and adolescents from a specialized out-patient clinic.

Methods: This cohort study with retrospective collection of data enrolled 150 patients ( 1 to 19 years old, 53\% males, $63 \%$ from Campinas, São Paulo, Brazil, and at mean age of 4.3 years when they started the weight gain). All patients were followed at the Obesity Out-Patient Clinic of the Clinical Hospital of Campinas University. Out of the 150 patients, 128 returned once and weight, height, body mass index (BMI) measurements and $\mathrm{Z}$ scores were obtained. Laboratory exams were colleted: hemoglobin, lipids, fasting glucose and fasting insulin. Glucose/insulin ratio was calculated. Patients were divided in two groups, if they returned or not to the clinic. Difference between both groups were analyzed regarding age, gender, city of origin, age when the weight gain started, acanthosis nigricans, weight, height, BMI Z score and proportion of altered laboratory exams. The difference in BMI Z score in relation to the first visit was also analyzed.

Results: Among the 128 patients, $57 \%$ continued to be followed in the clinic and 114 returned at least once. Comparing groups, patients from Campinas fail more to return. There were no differences in the clinical, anthropometric and laboratory variables. Both groups showed lower mean BMI $\mathrm{Z}$ score in the return visit compared to the first visit.

Conclusions: The obese children and adolescents from the follow-up clinic showed a reduction in the mean BMI
${ }^{1}$ Professor-assistente doutor do Departamento de Pediatria e médicos do Ambulatório de Crianças e Adolescentes Obesos do Hospital de Clínicas da Universidade Estadual de Campinas (HC-Unicamp), Campinas, SP, Brasil

${ }^{2}$ Professor-associado do Departamento de Pediatria e médico do Ambulatório de Crianças e Adolescentes Obesos do HC-Unicamp, Campinas, SP, Brasil
Endereço para correspondência:

Mariana Porto Zambon

Rua Botafogo, 151/491

CEP 13104-064 - Campinas/SP

E-mail: mzambon@fcm.unicamp.br

Recebido em: 29/1/2008

Aprovado em: 10/4/2008 
$\mathrm{Z}$ score similar to published data. The rate of dropping follow-up was high, but no variable could explain it, except for the patient origin.

Key-words: obesity, anthropometry; hematologic tests; child; adolescent.

\section{Introdução}

A alta prevalência mundial de obesidade em crianças e adolescentes, associada a seus fatores de risco e permanência na vida adulta, continua a ser um desafio para os profissionais de saúde ${ }^{(1-4)}$. Dentre as várias teorias a este respeito, acredita-se que a causa da obesidade se relaciona à adaptação do homem à vida moderna, o que repercute na criança em termos de diminuição da atividade física, aumento da ingestão alimentar e transtornos de comportamento cada vez mais precoces ${ }^{(1,2,5,6)}$. Apesar de novos conhecimentos quanto à fisiopatologia e a alternativas terapêuticas, clínicas e cirúrgicas, encontra-se pouco sucesso no acompanhamento destes pacientes $^{(2,7-9)}$. A necessidade da mudança do modo de vida por tempo indeterminado para se obter bons resultados é, provavelmente, o que dificulta o tratamento e faz com que os pacientes abandonem o acompanhamento ${ }^{(10,11)}$. Alguns estudos têm procurado identificar as causas do abandono, avaliando fatores diversos como altos valores de índice de massa corpórea (IMC), perda rápida de peso e utilização de planilha alimentar, entre outros ${ }^{(10,11)}$.

O objetivo deste trabalho foi analisar dois anos consecutivos do seguimento de crianças e adolescentes obesos em ambulatório especializado, que possui avaliação médica, nutricional, psicológica e de educador físico, e preconiza retornos a cada três meses.

\section{Métodos}

Estudo retrospectivo de acompanhamento dos pacientes matriculados no Ambulatório de Obesidade na Criança e Adolescente do Hospital de Clínicas da Universidade Estadual de Campinas (Unicamp), entre abril de 2005 e março de 2007. Foram incluídas todas as crianças e adolescentes atendidos com diagnóstico de obesidade, (ou seja, com IMC maior que o percentil 95 para idade e sexo, de acordo com as curvas do Centers for Disease Control and Prevention (CDC) $\left.{ }^{(12)}\right)$ e que fizeram pelo menos uma consulta neste período, independentemente da data da primeira consulta e da presença de outras doenças concomitantes.
Para a análise clínica, utilizaram-se dados relativos a idade, sexo, procedência e idade de início do ganho de peso. Medidas de peso e altura foram realizadas utilizando as técnicas descritas por Cameron ${ }^{(13)}$, com as quais calculou-se o IMC. Para as variáveis antropométricas (peso, altura e IMC), foram obtidos os respectivos escores $Z$, tendo como referência a curva do $\mathrm{CDC}^{(12)}$. A análise laboratorial foi feita de acordo com os exames solicitados na rotina do ambulatório, realizados habitualmente no Laboratório de Patologia Clínica do HC-Unicamp, com técnicas previamente padronizadas: hemograma, glicemia de jejum, insulina basal, triglicérides, colesterol total e frações. Considerou-se anemia de acordo com os níveis de hemoglobina padronizados por faixa etária pela Organização Mundial de Saúde $(\mathrm{OMS})^{(14)}$. Foram dados como alterados: relação glicemia/insulina $<7$; colesterol total $\geq 170 \mathrm{mg} / \mathrm{dL}$, LDL colesterol $\geq 130 \mathrm{mg} / \mathrm{dL}$, HDL colesterol $\leq 45 \mathrm{mg} / \mathrm{dL}$ e triglicérides $\geq 130 \mathrm{mg} / \mathrm{dL}^{(15)}$.

Os pacientes foram divididos em dois grupos: aqueles que mantiveram e os que perderam o seguimento, sendo o último composto pelos pacientes que não retornaram em até de cinco meses (normalmente o retorno é marcado após três meses). $\mathrm{Na}$ formação dos grupos, excluiram-se quatro pacientes por dificuldade técnica de obter as medidas de peso e altura (paraplegia) e 18 pacientes por terem sido vistos pela primeira vez no período final do estudo - portanto sem tempo para o retorno. Entre os grupos, foi analisada a diferença de idade, sexo, procedência, idade de início do ganho de peso, escores $Z$ de peso, altura e IMC, resultado dos exames laboratoriais, e média da diferença entre os escores $Z$ para aqueles com mais de um retorno.

Todos os dados foram computados em uma ficha clínica e analisados pelo programa Statistical Package for the Social Sciences (SPSS), versão 13.0. Por se tratar de estudo retrospectivo, a análise foi feita com base nos dados disponíveis. Para a comparação, foi usado o teste do qui-quadrado, o teste exato de Fischer ou o teste $t$, de acordo com a variável estudada. Os resultados foram considerados estatisticamente significantes quando $p<0,05^{(16)}$.

A presente investigação foi aprovada pelo Comitê de Ética e Pesquisa da Faculdade de Ciências Médicas da Unicamp, com dispensa do termo de consentimento livre e esclarecido.

\section{Resultados}

No período estudado, foram atendidos 150 pacientes com idade entre um e 19 anos, dos quais 77 (53\%) eram do sexo mascu- 
lino, a maioria (92-63\%) da região de Campinas, São Paulo, com média da idade no início de ganho de peso de 4,3 anos, variando de um a 13 anos. As medidas de peso, altura e IMC, assim como as análises laboratoriais iniciais, estão descritas na Tabela 1.

$\mathrm{Na}$ formação dos grupos, foram incluídos 128 pacientes, dos quais $73(57 \%)$ permaneceram em seguimento, sendo que 114 retornou pelo menos uma vez. O número de pacientes que retornou entre duas e oito vezes foi diminuindo progressivamente, sendo este número de $86,63,46,35,25$ e 18 , respectivamente. O abandono foi de $55(43 \%)$ pacientes; destes, 14 (25\%) já não compareceram no primeiro retorno e apenas três $(0,05 \%)$ deixaram de voltar após um ano. $\mathrm{O}$ intervalo de tempo entre cada um dos retornos variou de zero a oito meses e a média foi de 1,7 a 2,8 meses.

Não houve diferença entre os grupos em relação às variáveis clínicas (idade, idade de início, sexo, presença de acantose nigricans), antropométricas (escores $\mathrm{Z}$ de peso, altura e IMC) e laboratoriais (hemoglobina, glicemia de jejum, insulina basal, relação glicemia/insulina, triglicérides, colesterol total e frações), conforme Tabelas 2 e 3 . A comparação entre os grupos mostrou diferença estatisticamente significativa apenas em relação à pro- cedência dos pacientes, sendo que os residentes em Campinas abandonaram mais o ambulatório (Tabela 3).

No acompanhamento dos pacientes, observou-se queda da média do escore $Z$ do IMC em todos os retornos, nos dois grupos. A maior diminuição da média do IMC foi encontrada no grupo ainda em seguimento $(-0,12)$, no quarto retorno. A menor diminuição média $(-0,005)$ foi notada no grupo que abandonou o acompanhamento, na oitava consulta. Não houve diferença estatisticamente significativa entre os grupos na média de variação do escore $Z$ do IMC entre os retornos e a primeira consulta (Tabela 4).

\section{Discussão}

O ambulatório de crianças e adolescentes obesos do HC-Unicamp apresenta corpo clínico multiprofissional, com docentes e residentes de primeiro ano de Pediatria, nutricionista, educador físico e psicólogo. Rotineiramente, na primeira consulta, é realizado atendimento médico com solicitação de exames laboratoriais, orientação sobre hábitos de vida saudáveis e, em casos selecionados, uma avaliação nutricional. Nos retornos, faz-se avaliação médica, verifica-

Tabela 1 - Distribuição dos valores da média, desvio padrão, mínimo, $1^{\circ}, 2^{\circ}$ e $3^{\circ}$ quartil e máximo da idade, idade de início, peso, altura, IMC e exames laboratoriais à primeira consulta

\begin{tabular}{lrrrrrrrr}
\hline & $\mathbf{N}$ & Média & $\begin{array}{c}\text { Desvio } \\
\text { padrão }\end{array}$ & Mínimo & $\begin{array}{c}\mathbf{1}^{\mathbf{0}} \\
\text { Quartil }\end{array}$ & $\begin{array}{c}\mathbf{2}^{\mathbf{0}} \\
\text { Quartil }\end{array}$ & $\begin{array}{c}\mathbf{3}^{\mathbf{0}} \\
\text { Quartil }\end{array}$ & Máximo \\
\hline Idade (anos) & 150 & 10,5 & 3,4 & 1,0 & 8,7 & 11,0 & 13,0 & 19,0 \\
Idade de início (anos) & 129 & 4,3 & 3,1 & 1,0 & 1,0 & 4,0 & 7,0 & 13,0 \\
Peso (kg) & 146 & 72,0 & 25,6 & 19,9 & 54,4 & 72,0 & 88,5 & 146,0 \\
Altura (m) & 146 & 1,5 & 0,2 & 0,9 & 1,4 & 1,5 & 1,6 & 1,8 \\
IMC (kg/m $\left.{ }^{2}\right)$ & 146 & 31,7 & 6,2 & 21,1 & 26,8 & 30,9 & 35,8 & 58,5 \\
Hemoglobina (g/dL) & 102 & 13,4 & 1,0 & 10,8 & 12,7 & 13,5 & 14,1 & 15,7 \\
Glicemia (mg/dL) & 134 & 83,2 & 7,2 & 62,0 & 79,0 & 83,0 & 87,2 & 107,0 \\
Insulina (uUl/mL) & 99 & 17,7 & 13,4 & 3,8 & 8,7 & 14,0 & 22,5 & 82,2 \\
Colesterol total (mg/dL) & 132 & 162,3 & 32,4 & 118,0 & 144,2 & 160,5 & 176,7 & 326,0 \\
Triglicérides (mg/dL) & 131 & 124,2 & 140,1 & 44,0 & 73,0 & 99,0 & 141,0 & 1599,0 \\
LDL-colestrol (mg/dL) & 123 & 96,4 & 30,2 & 33,0 & 79,0 & 94,0 & 45,0 & 208,0 \\
HDL-colesterol (mg/dL) & 125 & 46,0 & 30,2 & 26,0 & 39,0 & 45,0 & 51,5 & 79,0 \\
Relação glicemia/insulina & 99 & 7,3 & 1,0 & 1,3 & 3,8 & 6,1 & 9,9 & 20,3 \\
\hline
\end{tabular}

Tabela 2 - Distribuição dos valores mínimo, máximo, média e desvio padrão (dp) das variáveis clínicas e antropométricas no grupo de pacientes que abandonou e continuou o seguimento

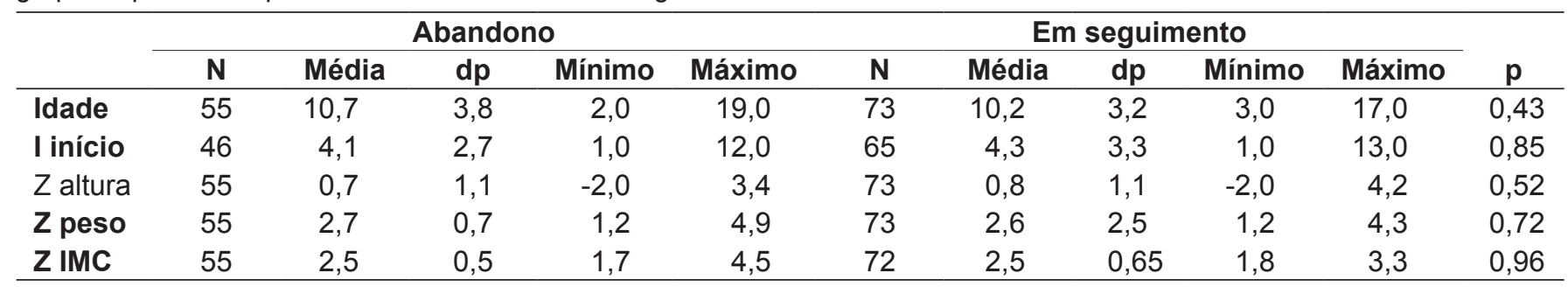

Idade em anos; I início: idade do início do ganho de peso em anos; Z: escore Z; $p$ : relativo ao teste $t$ entre os grupos. 
Tabela 3 - Prevalência de sexo, procedência, acantose nigricans e alteração dos exames laboratoriais dividido entre o grupo de pacientes que abandonou e continuou o seguimento

\begin{tabular}{|c|c|c|c|c|}
\hline & & Abandono & Em seguimento & $p$ \\
\hline \multirow[t]{2}{*}{ Sexo } & Feminino & 26 & 35 & 0,54 \\
\hline & Masculino & 29 & 38 & \\
\hline \multirow[t]{2}{*}{ Procedência } & Campinas & 30 & 19 & 0,001 \\
\hline & Região & 25 & 54 & \\
\hline \multirow[t]{2}{*}{ Acantose nigricans } & Ausente & 10 & 22 & 0,392 \\
\hline & Presente & 23 & 40 & \\
\hline \multirow[t]{2}{*}{ Glicemia } & Normal & 45 & 73 & 0,387 \\
\hline & Elevada & 1 & 0 & \\
\hline \multirow{2}{*}{ Insulina } & Normal & 20 & 31 & 0,233 \\
\hline & Elevada & 21 & 22 & \\
\hline \multirow[t]{2}{*}{ Colesterol total } & Normal & 31 & 50 & 0,377 \\
\hline & Elevado & 16 & 21 & \\
\hline \multirow[t]{2}{*}{ Triglicérides } & Normal & 38 & 49 & 0,135 \\
\hline & Elevado & 9 & 21 & \\
\hline \multirow[t]{2}{*}{ LDL } & Normal & 36 & 62 & 0,484 \\
\hline & Elevado & 5 & 7 & \\
\hline \multirow[t]{2}{*}{ HDL } & Normal & 27 & 26 & 0,007 \\
\hline & Elevado & 16 & 44 & \\
\hline \multirow[t]{2}{*}{ Relação glicemia/insulina } & Normal & 15 & 24 & 0,237 \\
\hline & Diminuída & 26 & 28 & \\
\hline
\end{tabular}

p: relativo ao $\chi^{2}$ ou ao teste Exato de Fisher.

Tabela 4 - Evolução do escore Z de IMC entre os retornos, dividido entre o grupo de pacientes que abandonou e continuou seguimento

\begin{tabular}{lccccccccccc}
\hline & \multicolumn{9}{c}{ Abandono } & \multicolumn{4}{c}{ Em seguimento } & \multicolumn{3}{c}{ Teste $t$} \\
$\mathbf{Z}$ IMC & $\mathbf{N}$ & Média & $\mathbf{d p}$ & Mínimo & Máximo & $\mathbf{N}$ & Média & dp & Mínimo & Máximo & $\boldsymbol{p}$ \\
\hline $2^{\circ}-1^{\circ}$ & 42 & $-0,06$ & 0,15 & $-0,95$ & 0,08 & 71 & $-0,04$ & 0,07 & $-0,37$ & 0,14 & 0,65 \\
$3^{\circ}-1^{\circ}$ & 29 & $-0,05$ & 0,07 & $-0,24$ & 0,08 & 56 & $-0,06$ & 0,16 & $-1,03$ & 0,11 & 0,55 \\
$4^{\circ}-1^{\circ}$ & 16 & $-0,05$ & 0,09 & $-0,34$ & 0,04 & 46 & $-0,12$ & 0,39 & $-2,57$ & 0,21 & 0,49 \\
$5^{\circ}-1^{\circ}$ & 11 & $-0,06$ & 0,15 & $-0,48$ & 0,07 & 36 & $-0,10$ & 0,17 & $-0,59$ & 0,26 & 0,42 \\
$6^{\circ}-1^{\circ}$ & 7 & $-0,08$ & 0,18 & $-0,48$ & 0,05 & 28 & $-0,11$ & 0,22 & $-0,85$ & 0,26 & 0,72 \\
$7^{\circ}-1^{\circ}$ & 5 & $-0,10$ & 0,24 & $-0,52$ & 0,07 & 20 & $-0,11$ & 0,25 & $-0,84$ & 0,27 & 0,91 \\
$8^{\circ}-1^{\circ}$ & 2 & $-0,005$ & 0,06 & $-0,05$ & 0,04 & 16 & $-0,11$ & 0,27 & $-0,75$ & 0,39 & 0,58 \\
\hline$Z$
\end{tabular}

$Z I M C 2^{\circ}-1^{\circ}$ : média da diferença do escore $Z$ do entre a segunda e a primeira consulta e assim sucessivamente.

se o resultado dos exames, realiza-se consulta com educador físico que programa um teste físico e inicia-se a orientação alimentar por nutricionista; quando necessário, solicita-se avaliação da psicologia. Este ambulatório tem como objetivo atuar por meio de intervenções de conscientização dos pacientes e seus familiares em relação aos riscos da doença e suas conseqüências, tratamento das comorbidades, orientações nutricionais e aumento da atividade física.

O ambulatório já existe há dois anos e avaliou 150 crianças e adolescentes com diagnóstico de obesidade. Desde a primeira consulta, externa-se a grande preocupação com o risco de evolução para complicações cardiovasculares, pois 
os pacientes apresentam IMC bastante elevado, presença de acantose nigricans e alterações laboratoriais que caracterizam resistência à insulina sem descompensação diabética, além de alterações do metabolismo lipídico, como demonstrado em estudo preliminar ${ }^{(17)}$. Para avaliar o risco dos pacientes, pode-se agrupá-los com o diagnóstico de síndrome metabólica - o que se optou por não realizar, avaliando-se os fatores de risco separadamente. Isto se deve ao fato desta síndrome ser descrita em adultos e adaptada para crianças e adolescentes, podendo incluir pacientes com alterações discretas de alguns parâmetros e excluir pacientes com risco elevado, que apresentem apenas uma variável de síndrome ${ }^{(18,19)}$.

No acompanhamento destes pacientes, foram avaliados até oito retornos no período, com tempo médio entre estes de 1,8 no primeiro a 2,4 meses no último retorno. O número de pacientes nos retornos diminuiu progressivamente devido ao abandono do seguimento e por uma limitação do estudo, que incluiu pacientes novos no decorrer de todo o período analisado. Neste trabalho, $43 \%$ abandonaram o acompanhamento, dado semelhante ao observado em projeto norte-americano randomizado para o tratamento de crianças e adolescentes obesos com apoio familiar, orientações de atividade física e aconselhamento de modo de vida, que evidenciou seguimento de $60 \%$ após seis meses e de $53 \%$, após um ano, com perda de acompanhamento principalmente no grupo cuja intenção era a planilha alimentar ${ }^{(11)}$. Projeto longo, de cinco anos, que avaliou alterações de comportamento e distúrbios alimentares em adolescentes, também apresentou nível de abandono de $23 \%$ no primeiro contato, sendo que $53 \%$ dos pacientes finalizaram a pesquisa ${ }^{(20)}$. Tanas et $a^{(21)}$ propõem telefonar periodicamente para diminuir a falta de seguimento.

Neste trabalho, na tentativa de buscar indicadores para o alto índice de abandono, os pacientes foram divididos em dois grupos: os que perderam o seguimento e aqueles que mantiveram o acompanhamento. A única variável que apresentou diferença estatisticamente significativa foi a procedência do paciente. Inversamente ao esperado, os pacientes da cidade de Campinas tiveram maior nível de abandono - fato para o qual não temos explicação plausível.

Não foi observada diferença em nenhuma outra variável clínica (idade, sexo, idade de início), antropométrica (escores $\mathrm{Z}$ de peso, altura e IMC iniciais e suas evoluções) ou laboratorial (anemia, glicemia de jejum, resistência à insulina, alterações de triglicérides, colesterol e frações) associada a esta atitude. Denzer et $a l^{(10)}$ notaram que, em crianças e adolescentes, valores mais altos do escore $\mathrm{Z}$ de IMC estavam associados ao abandono do seguimento. Entretanto, a resposta obtida inicialmente ao tratamento proposto, no que se refere ao escore $\mathrm{Z}$ do IMC, não interferiu na aderência ao seguimento. Braet ${ }^{(22)}$ demonstrou, em um grupo de crianças entre sete e 17 anos, que as mais gordas, as mais velhas e as que obtiveram perda de peso mais precoce apresentaram melhores resultados em dois anos; o oposto ocorreu com aquelas com distúrbios alimentares.

Neste estudo, apesar do grande número de abandonos - incluindo o fato de que $25 \%$ já não comparecerem no primeiro retorno -, a evolução da média do escore $Z$ do IMC diminuiu em relação à primeira consulta em todos os retornos, analisando todos os pacientes ou dividindo-os de acordo com perda ou não de seguimento. A queda da média do escore $\mathrm{Z}$ do IMC aumentou progressivamente no grupo em seguimento até o quarto retorno, apresentando-se estável a partir de então. A comparação destes dados com outros estudos é difícil, devido às diferentes metodologias empregadas, incluindo a faixa etária, as características de intervenção, o tempo de estudo e as unidades de medida. Estudo de revisão bibliográfica americano avaliou 22 trabalhos, entre 1996 e 2004 - a maioria com acompanhamento de crianças e adolescentes obesos por curto tempo. Esta revisão indica resultados discretos de melhora do IMC ou a manutenção do mesmo ${ }^{(23)}$.

Trabalho preventivo inglês com escolares sadios de sete a 11 anos, randomizados em grupo controle e de tratamento, no qual o objetivo principal foi reduzir a ingestão de bebidas carbonatadas e acrescentar uma hora de educação física na escola por um ano, mostrou aumento no escore $\mathrm{Z}$ do IMC de 0,1 no grupo controle e queda de 0,01 no grupo tratado. Na continuação do mesmo estudo, após três anos, houve aumento do sobrepeso em ambos os grupos, com reversão da melhora observada nos primeiros 12 meses $^{(24)}$. Nos trabalhos com maior duração, observa-se que, mesmo que ocorra perda de peso, os pacientes não deixam de ser obesos ${ }^{(11,25)}$.

A literatura ainda é controversa em como considerar positivo o resultado do tratamento da obesidade, independente da faixa etária. Em adultos, acredita-se que a perda de $10 \%$ do peso pode acarretar melhora das comorbidades. Em relação às crianças e aos adolescentes, um consenso internacional de especialistas sugere que as expectativas devam ser individualizadas. Mas, qualquer diminuição no escore $\mathrm{Z}$ do IMC deve ser considerado como um bom resultado terapêutico, enquanto as metas ideais a serem atingidas não forem determinadas ${ }^{(5)}$. 
Em países desenvolvidos, ao se avaliar adultos com doenças crônicas, constatou-se dificuldade em manter o seguimento dos mesmos. Para a obesidade, a literatura tem mostrado trabalhos com pequenos intervalos de observação, às vezes com intervenção especializada e bons resultados durante esse período, mas com uma resposta desanimadora em longo prazo, sugerindo a necessidade de esquemas de conscientização para mudanças duradouras nos hábitos de vida $^{(2)}$ e, em caso de crianças e adolescentes, deve-se acrescentar o apoio familiar. Mesmo sabendo da dificuldade em obter bons resultados com esta doença, pois requer mudanças no ambiente que a propicia, e da falta de padronização no acompanhamento dos pacientes, os resultados obtidos neste estudo são comparáveis aos da literatura. Este achado faz com que continuemos o atendimento, aprimorando-o e estimulando a busca de mecanismos para manter o paciente no ambulatório. Diante gravidade do problema, não se pode esquecer de salientar a importância do diagnóstico precoce da obesidade e, principalmente, estimular medidas preventivas desde a puericultura, com hábitos alimentares e de atividade física saudáveis.

\section{Agradecimentos}

A toda equipe de profissionais que atua no Ambulatório de Crianças e Adolescentes Obesos do HC-Unicamp.

\section{Referências bibliográficas}

1. WHO. Obesity: preventing and managing the global epidemic - report of a WHO consultation on obesity, WHO/NUT/NCD/98.1. Geneva: World Health Organization; 1998.

2. Zwiauer KF. Prevention and treatment of overweight and obesity in children and adolescents. Eur J Pediatr 2000; 159 (Suppl 1):S56-68.

3. Wang Y, Monteiro C, Popkin BM. Trends of obesity and underweight in older children and adolescents in the United States, Brazil, China, and Russia. Am J Clin Nutr 2002;75:971-7.

4. Philippas NG, Lo CW. Childhood obesity: etiology, prevention, and treatment. Nutr Clin Care 2005;8:77-88.

5. Styne DM. Childhood and adolescent obesity. Prevalence and significance. Pediatr Clin North Am 2001;48:823-54.

6. Dorsey KB, Wells C, Krumholz HM, Concato JC. Diagnosis, evaluation, and treatment of childhood obesity in pediatric practice. Arch Pediatr Adolesc Med 2005; 159:632-8.

7. Tsiros MD, Sinn N, Coates AM, Howe PR, Buckley JD. Treatment of adolescent overweight and obesity. Eur J Pediatr 2008;167:9-16.

8. Speiser PW, Rudolf MC, Anhalt H, Camacho-Hubner C, Chiarelli F, Eliakim A et al. Childhood obesity. J Clin Endocrinol Metab 2005;90:1871-87.

9. Maryon-Davis A. Weight management in primary care: how can it be made more effective? Proc Nutr Soc 2005;64:97-103.

10. Denzer C, Reithofer E, Wabitsch M, Widhalm K. The outcome of childhood obesity management depends highly upon patient compliance. Eur J Pediatr 2004;163:99-104.

11. Savoye M, Shaw M, Dziura J, Tamborlane WV, Rose P, Guandalini C et al. Effects of a weight management program on body composition and metabolic parameters in overweight children: a randomized controlled trial. JAMA 2007;297:2697-704.

12. $\mathrm{CDC}$ (Centers for Disease Control and Prevention) [homepage on the Internet]. 2000 CDC growth charts: United States NCHS: BMI growth charts [cited 2008 Apr 4]. Available from: http://www.cdc.gov/growthcharts/
13. Cameron N. The methods of auxological anthropometry. In: Falkner F, Tanner JM, editors. Human growth. $1^{\text {st }}$ ed. New York: Plenum Press; 1978. p. 53-90.

14. World Health Organization [homepage on the Internet]. Iron deficiency anaemia: assessment, prevention, and control [cited 2008 Apr 4]. Available from:http:// who.int/nutrition/publications/en/ida_assessment_prevention_control.pdf

15. Sociedade Brasileira de Cardiologia. I diretriz de prevenção da aterosclerose na infância e na adolescência. Arq Bras Cardiol 2005;85:S2-36.

16. Montgomery DC. Design and analysis of experiments. $3^{\text {rd }}$ ed. New York: Wiley \& Sons; 1991

17. Zambon MP, Antonio MA, Mendes RT, Barros Filho AA. Características clínicas e laboratoriais de crianças e adolescentes obesos. Rev Paul Pediatr 2007;25:27-32.

18. Reaven GM. The metabolic syndrome: is this diagnosis necessary? Am J Clin Nutr 2006;83:1237-47.

19. Goodman E, Daniels SR, Meigs JB, Dolan LM. Instability in the diagnosis of metabolic syndrome in adolescents. Circulation 2007;115:2316-22.

20. Haines J, Neumark-Sztainer D, Eisenberg ME, Hannan PJ. Weight teasing and disordered eating behaviors in adolescents: longitudinal findings from Project EAT (Eating Among Teens). Pediatrics 2006;117:e209-15.

21. Tanas R, Marcolongo R, Pedretti S, Gilli G. A family-based education program for obesity: a three-year study. BMC Pediatr 2007;7:33.

22. Braet $C$. Patient characteristics as predictors of weight loss after an obesity treatment for children. Obesity 2006;14:148-55.

23. Whitlock EP, Williams SB, Gold R, Smith PR, Shipman SA. Screening and interventions for childhood overweight: a summary of evidence for the US Preventive Services Task Force. Pediatrics 2005;116:e125-44.

24. James J, Thomas P, Kerr D. Preventing childhood obesity: two year followup results from the Christchurch obesity prevention programme in schools (CHOPPS). BMJ 2007;335:762-5.

25. Epstein LH, Valoski A, Wing RR, McCurley J. Ten-year follow-up of behavioral, family-based treatment for obese children. JAMA 1990;264:2519-23. 